\section{Dyeing and Finishing Machinery}

Takashi Yamazaki* and Hiroshi Yokoyama**

*Professor, Kyoto College of Education

**Consulting Engineer

\section{Introduction}

Nowadays, whole textile industry tends to full automation owing to the development of electronics, and will enter the era of Factory Automation (FA) in the near future. In the field of dyeing and finishing too, countermeasures to small quantity production with many sorts and deversification, efforts to high-grade and specialization, are making high progress. Our main purpose of observing the 4th OTEMAS is to inspect the ability of realizing Factory Automation in dyeing and finishing in the future.

The main themes of the 2nd and the 3rd OTEMAS were energy saving and automation, respectively. At the 4th OTEMAS, gradual and solid efforts towards Factory Automation were found in many displayed machines, although Factory Automation in dyeing and finishing appears to be more difficult than in spinning and weaving because of including many complicated problems related to chemistry, thermodynamics and hydrodynamics. Interesting machines or engineering systems observed at the 4th OTEMAS will be presented in the following.

\section{Yarn Dyeing Machines}

In the field of package dyeing, many machines or systems aiming at high performance and high productivity such as Hisaka Works' "Model LLC" were observed. FA systems possible in the present stage were introduced into those machines.

"LLC plant total production system" of Hisaka Works is characterized by rapid dyeing process and low liquor ratio dyeing: for instance, necessary total time for cotton reactive dyeing is decreased to one half of conventional dyeing machines. System for efficiently handling small quantity with many sorts was displayed as shown in Fig. 1. Panel display for "operaterless system" (Hisaka

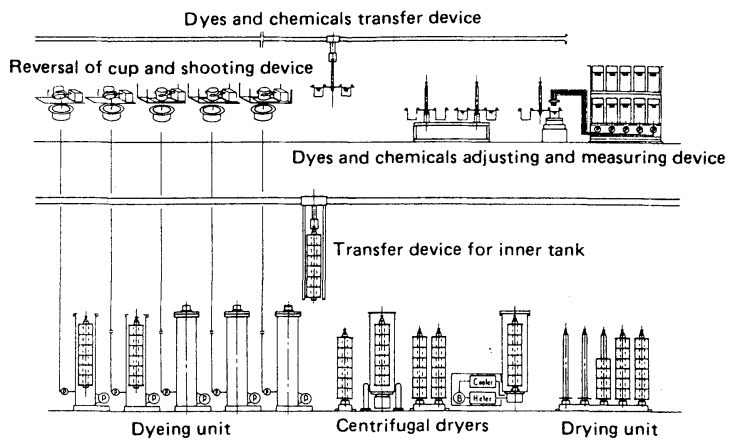

Fig. 1 Flow sheet for small quantity dyeing system of Hisaka

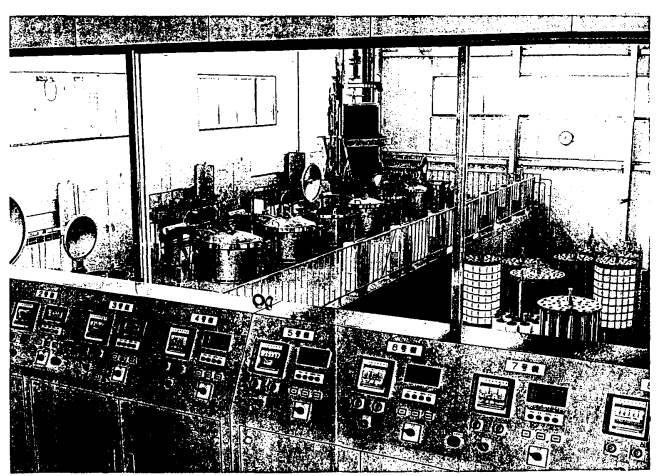

Fig. 2 Panel display for operaterless system (Hisaka)

\section{Works) is shown in Fig. 2.}

High pressure, high temperature cheese dyeing machine of Suzuki Manufacturing Co. (HCDE-1) aims at reducing dyeing time and cost by low liquor-ratio. Full automatic dyeing system was also displayed by this company. Similar equipment of Tong Wu Precision Machinery Co. (Taiwan) was observed.

As package dryers, "micro-wave cheese dryer model HFD" and "pair dryer model UEFC-A" (Hisaka Works) were observed (Fig. 3). None of the former type cheese
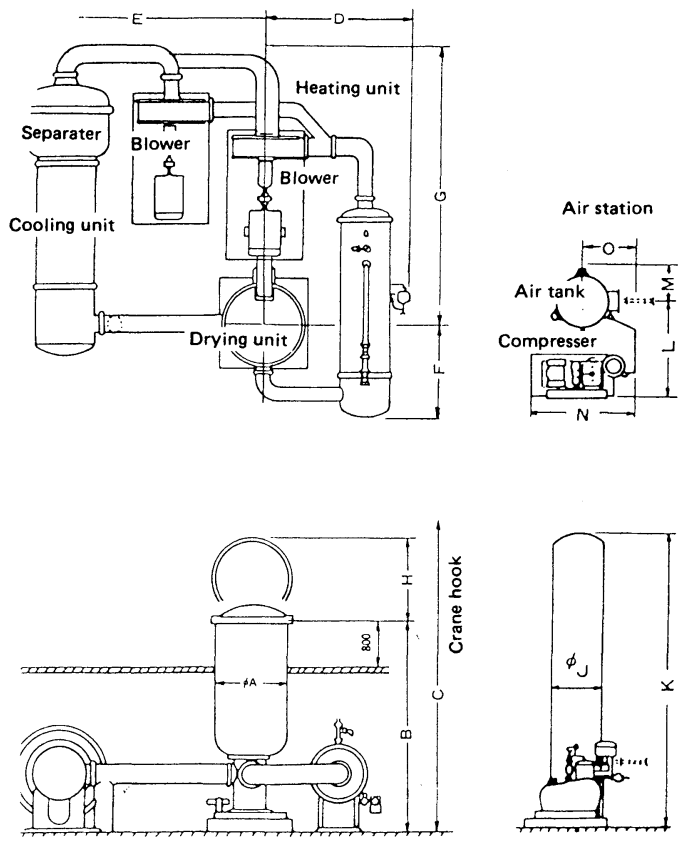

Fig. 3 Rapid cheese pair dryer (Hisaka)

dryer was found at the 3rd OTEMAS. The latter minimizes the waste time by combining large and small lots.

Spray type hank dyeing machine "Somemaru" of Suzuki Manufacturing Co. was connected with five dyeing units of low liquor ratio, and seemed suitable for small quantity production. 


\section{Preparation for Fabric}

\subsection{Fabric joiners}

"Cloth Tacker" of Yachida was displayed by C. Itoh Texmac Co. as a full automatic fabric joiner (Fig. 4).

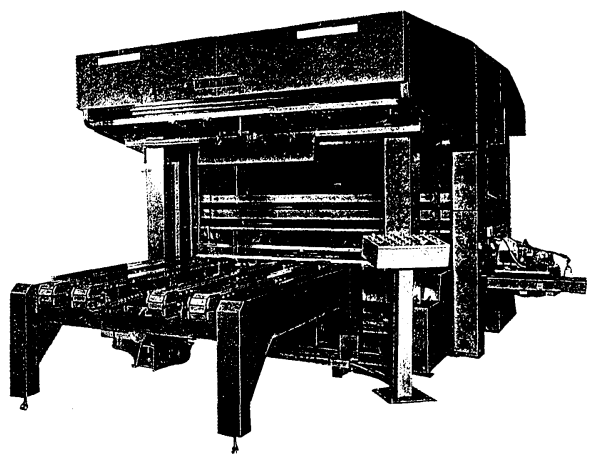

Fig. 4 Cloth tacker of Yachida - C. Itoh Texmac Co.

The machine is fitted with a overlock sewing machine, and necessary time was only $40-50 \mathrm{sec}$ for one joint having width of $900-2,000 \mathrm{~mm}$ or diameter of $70-$ $200 \mathrm{~mm}$.

"Tack-Robo Model ST-1" of Seiren Electronics Co., a device for automatic doubling and sewing of woolen fabric in preparing dyeing and finishing process, was observed. Its specification was as follows - cloth width

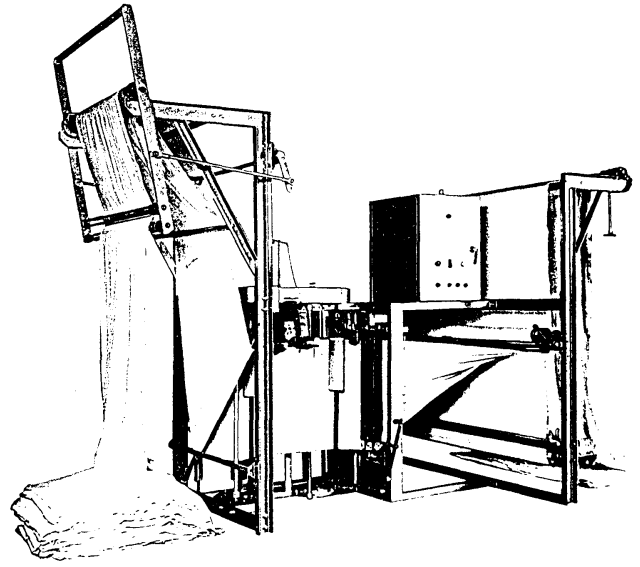

Fig. 5 Tack-Robo-ST-1 of Seiren Electronics

$1,200-1,800 \mathrm{~mm}$, speed $10-40 \mathrm{~m} / \mathrm{min}$, stitch $35-$ $75 \mathrm{~mm}$ and alignment accuracy less than $5 \mathrm{~mm}$ (Fig. 5).

\subsection{Milling-scouring machines}

C. Itoh Texmac Co. displayed Serracant's MillingScouring Machine BD-1650. It was equipped with independent adjusting devices and digital metering devices, and was operated semiautomatically without any defects such as holes or creases (Fig. 6).

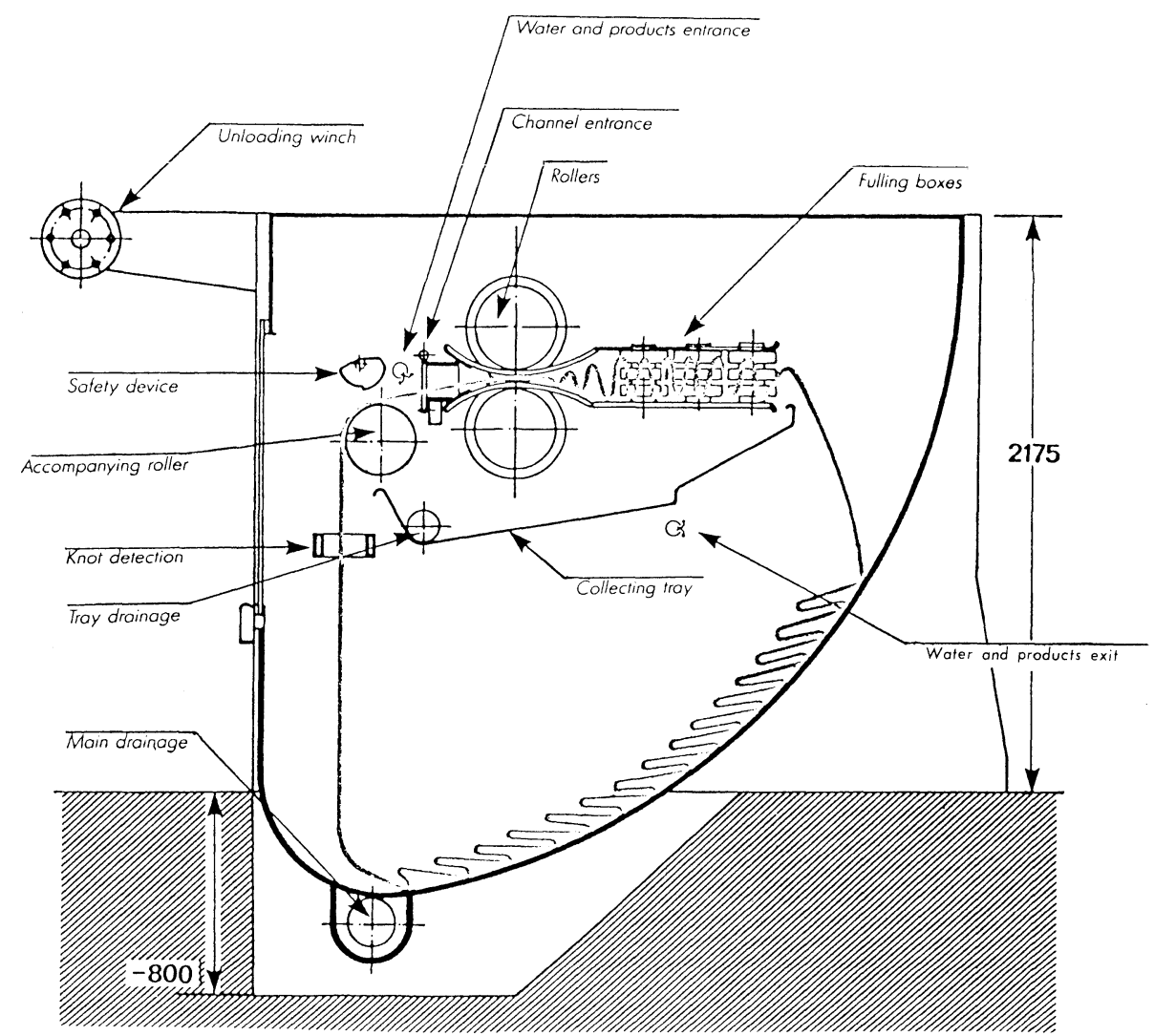

Fig. 6 BD-1650 Milling-Scouring of C. Itoh Texmac Co. 


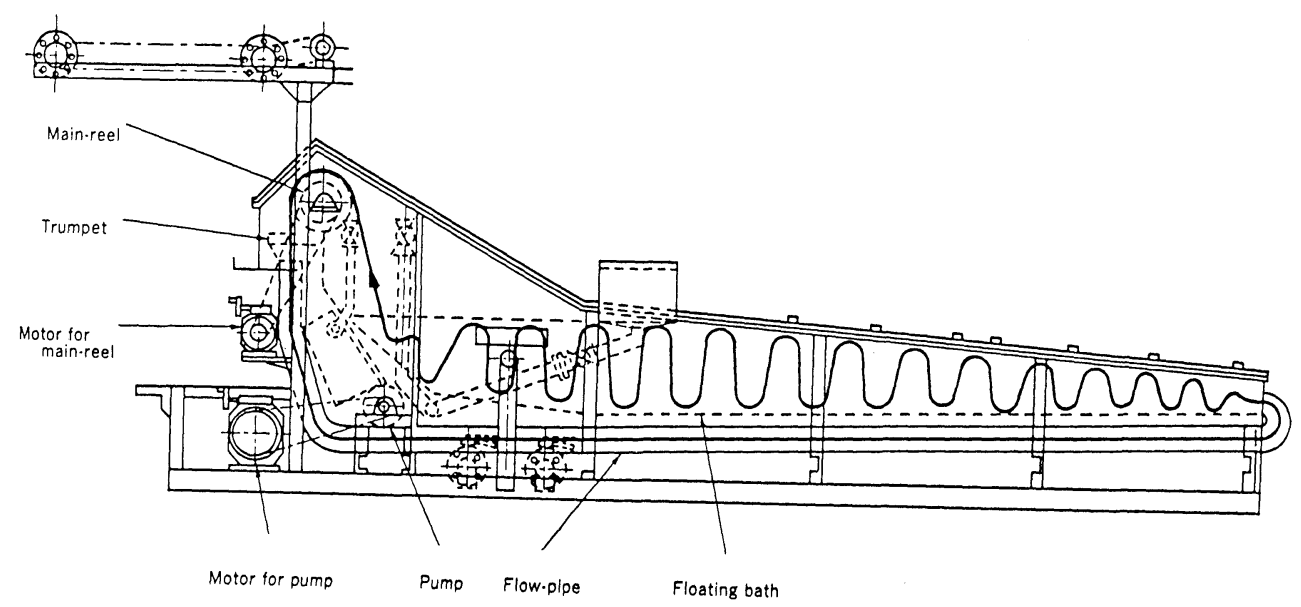

Fig. 7 Mechanism of jet dyeing machine "Dash Line" of Oshima Machinery Co.

\subsection{Caustic soda treatment units for softening}

None of interesting machines were observed other than "Apollotex" of Ichikin Ltd.

\section{Dyeing Machines for Fabrics}

As far as continuous dyeing machines are concerned, there was nothing worthwhile to be explained. Batch dyeing machine for fabrics tends towards low liquor ratio and versatility similarly to package dyeing machines. Considering FA in the future, automation or central control system was included. Many of them showed versatility for various fibers and for woven and knitted fabrics.

\subsection{Jet dyeing machines}

Normal pressure jet dyeing machine "Dash Line" of Oshima Machinery Co. attracted attention of many visitors, by combining the continuous flow-batch technique (type SE-C) (Fig. 7). The unit widens the fabric at the flow pipe exit in the balloon form, and so the position of wrinkle always changes. Therefore the unit is suitable for easy-to-wrinkle fabrics such as cotton or polyester/cotton knits. As similar normal pressure jet dyeing machines, Nissen's "Swing-Ace", Hisaka's

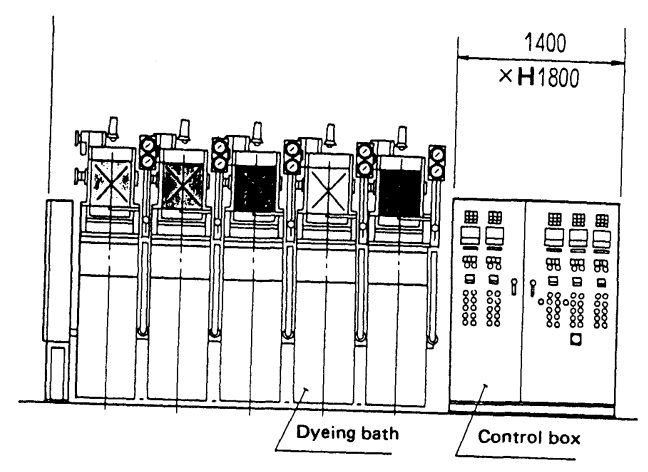

Fig. 8 "Multi-Color Swing-Ace" of Nissen
CUT-AM, Tong Wu's "Model TWRU-NPL" were displayed. "Multi-color Swing-Ace" attracted attention by its suitability for small quantity with many sort production (Fig. 8).

In the field of high pressure dyeing machines, Hisaka's "Circular CUT-RZ" was hopeful as a low liquor type. As a similar machine, Nissen's "Rapid Uniace" series and Tong Wu's "Model TWRU-HA" were observed. Those high pressure jet dyeing machines were characterized by high speed fabric circulation and soft touch of fabrics.

\section{Auxiliary Machines for Color Matching}

According to the increased need for small quantity with many sorts, full automatic color matching systems were displayed by many manufacturers or trading companies at the 4 th OTEMAS too.

\subsection{Computer color mathing systems (CCM)}

In order to increase efficiency and accuracy, numbers of color measuring devices connected with color kitchen system were observed at the 4th OTEMAS.

Among many color measuring devices, Minolta's spectro-photometers were utilized in many devices together with Macbeth's MS-2020. Both "Spectro Color Difference Meter CR-200" and "Spectro Color Machine CM-1000" of Minolta were protable. They need small measuring samples of only $8-11 \mathrm{~mm}$ in diameter.

As an example of improved performance and versatility, Nippon Kayaku Co. displayed three types of computer color matching systems "Comsek-III, -101 and -201 ". They were connected with computer color kitchen system "Kayalibra" series to rationalize color control in dye house. Among Kayalibra series, K-2 type drastically reduces the dye liquor preparation time by using its "robot hand" system and "four head simultaneous injection systems" (Fig. 9).

Kurabo Co. displayed automatic color kitchen "Aukitchen" series, -60 of volumetric type and -LA of weighing type, and other related color control systems. 

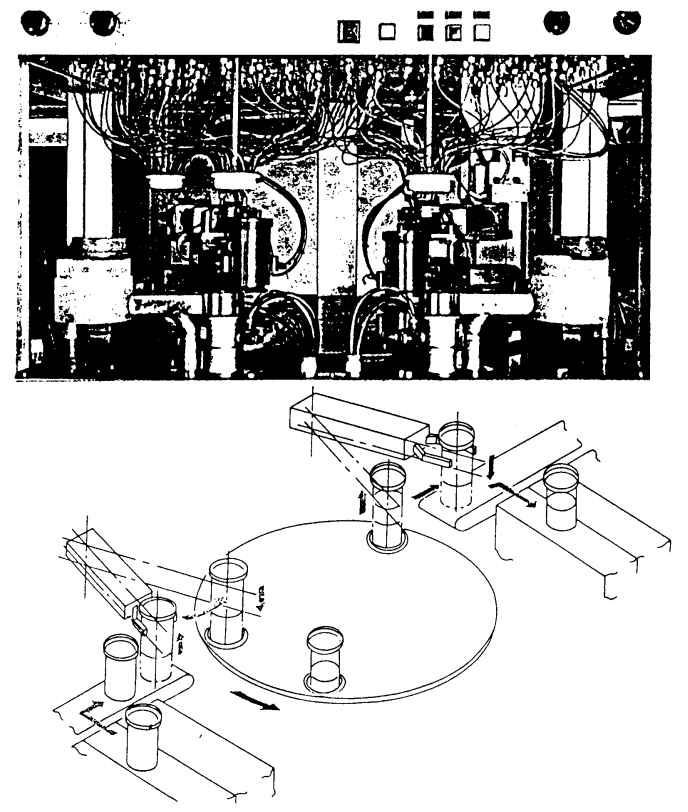

Fig. 9 "Kayalibra K-2" and "Robot hand" system of Nippon Kayaku

CCM Systems of EXCOM and Sedo were also worthy of note.

\subsection{Dyeing program controllers}

Oshima Machinery's "FA-II" — a programable controller exclusive for dyeing machine - was displayed. Its interactive mode makes setting easy even for a beginner on programmable digital controllers. The fine and wide display gives a clear view in setting-up dyeing. This controller was developed to apply it to Oshima's "Dash-Line", and will render a great service towards FA.

New prototype of P-series computer controller for dyeing machines of Sedo Textile Computer Co. was observed (Fig. 10, 11). Older P-series of this controller was displayed as a product of Sehermuly Elektronik
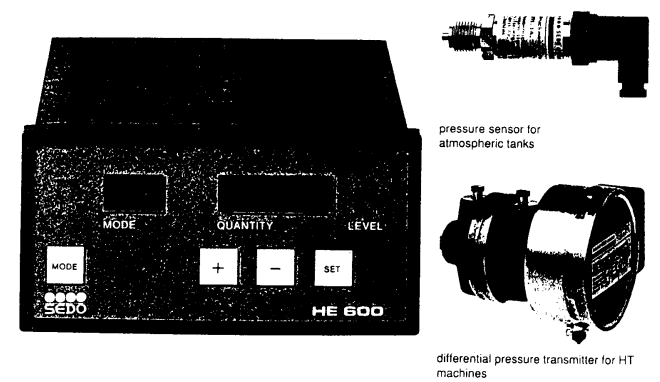

Fig. 11 "Niveaumat-HE600" and its accessories of Sedo Textile Computer

Co. at the 3rd OTEMAS.

\section{Printing Machines for Fabric}

\subsection{Automatic printing thickner preparing system}

"Ichinose Colorway" of Toshin Industry was displayed again like at the 3rd OTEMAS. The merit of liquid type reactive dyes of Ciba Geigy Co. can be brought about by using "Colorway". A special container suitable for carrying and storing liquid reactive dye was developed for this purpose by Ciba Geigy (Fig. 12).

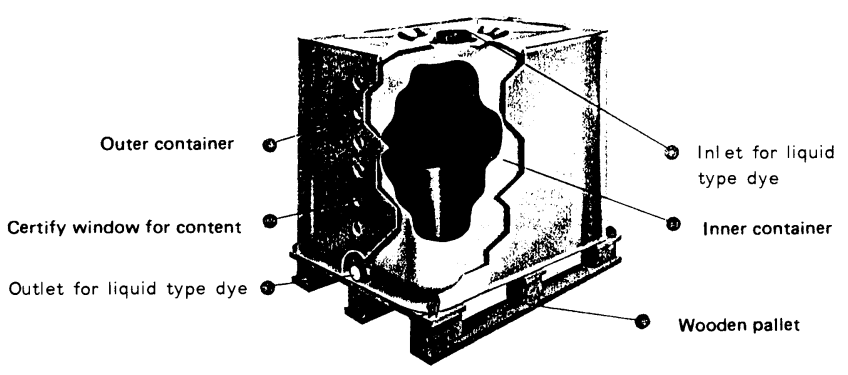

Fig. 12 Special container

\subsection{Printing machines}

Toshin Industry's “Ichinose-7000”, which was dis-

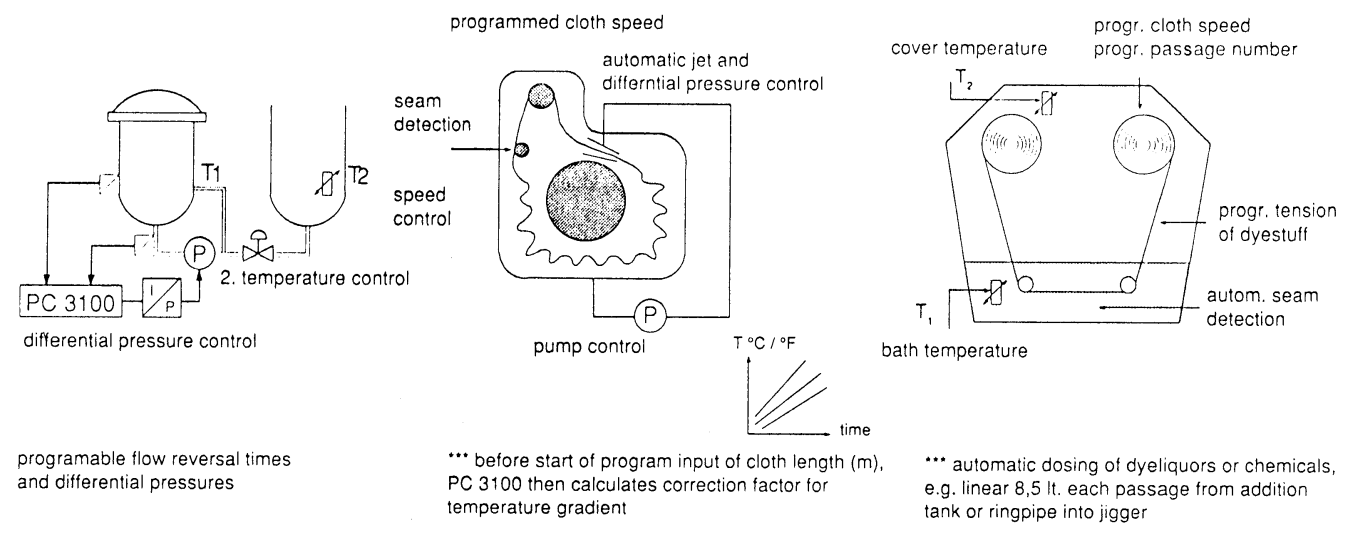

Fig. 10 Computer controller of yarn dyeing, jet, HTjigger by PC-3100 of Sedo Textile Computer 
played at the 3rd OTEMAS, was observed as a representative of flat screen printing machines. It was characterized by the print belt driving mechanism by DC motors, variable squeezing speed, and screen lifting system of good release from printing fabric. Similar mechanisms were found in "Rokki Model RE-1000" and "RE-1500" of Uenoyama Kiko Co. Both of them were paid much attention to their hot flue drying zones.

In the field of rotary screen printing machines, Toshin's "Ichinose-MR-600" and Uenoyama's "Dia Rota II" attracted attention, though the progress from the 3rd OTEMAS was not clear.

Uenoyama's T-shirt printing machine "Plasmavenus" was noticed as a machine suitable for more manifold order (Fig. 13).

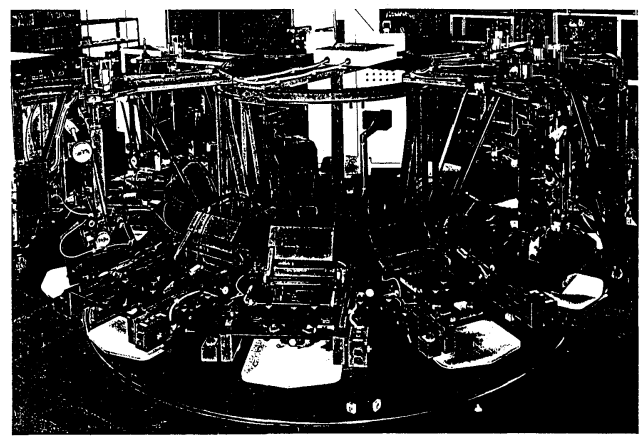

Fig. 13 "Plasmavenus" T-shirts printing machine of Uenoyama

\section{Heat Setting Machines and Dryers}

Ichikin Co.'s "Victex Stenter" and Hirano Tecseed's "Cleantec Stenter" (Fig. 15) were noticed by their automation devices. The former was characterized by modified

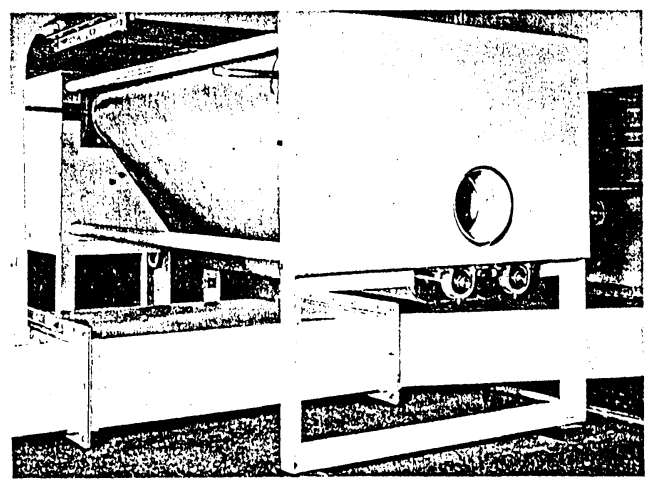

Fig. 14 Automatic air-filter cleaning device

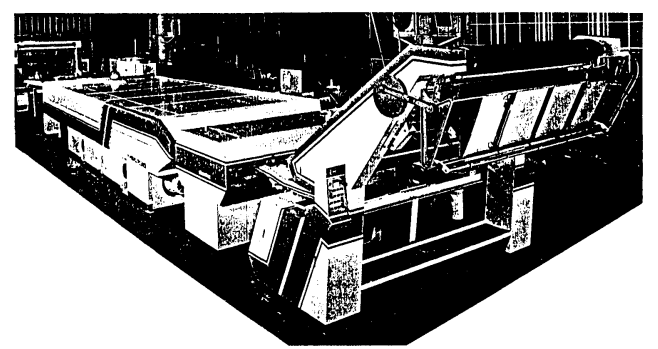

Fig. 15 Cleantec stenter of Hirano Tecseed clips and conveyer chains itself completely clean. Its automatic air-filter cleaning device is shown in Fig. 14.

Suction drum dryers were displayed by two manufacturers, Fleissner GmbH and Gaston County Co., Ltd.

\section{Washing Machines}

Uenoyama Kiko's “Jet-Stream-Soaper” was improved by introducing waste stream and high frequency vibration into the vapor phase of the enclosed vessel, therefore good wash-off effect and energy saving were obtained.

Suzuki Manufacturing Co.'s WMH-1 attracted attention as a high pressure high temperature drum washer.

\section{Other Machines and Systems}

Among other machines and systems, numbers of automatic measuring devices for FA aiming at stability of quality, improvement of productivity were observed.

\subsection{Continuous measuring devices}

Continuous moisture content measuring device of nontouch IR-type or electric conductivity type were displayed by Mate Science Co. They are useful for monitoring fabric condition in dryers, especially in drum dryers. As these devices can inspect fabric joint and width too, combination of these functions should contribute to realize FA.

\subsection{Rolls for padders and calenders}

"Uron H roll" for high pressure squeezing, "Elaglass RE" for calendering, and non-adhesive silicone rubber roll were displayed by Kinyosha Co.

\subsection{Treating machines for inorganic fibers}

New-type thread treating devices for impregnating threads made of carbon fibers or glass fibers were displayed. These were payed much attention as machines fit for treating new industrial fibers in the near future.

\section{Conclusion}

As mentioned before, the step towards FA in dyeing and finishing industry may be slower than that in spinning and weaving. But automation in specific devices or machines appears to have been remarkably progressed. In the 4th OTEMAS, conversion from energy saving to automation for countermeasures to meet small quantity production of many lots was seen apparant. At the next OTEMAS total FA will become the main theme instead of specific device automation in dyeing and finishing field too. 